\title{
Ueber eine örtliche Giftwirkung des Phenylhydroxylamin.
}

Ein Beitrag zar Kenntnis der Verbreitung von Giften durch die Hautlymphbahnen.

Von L. Lewin.

Ueber die resorptiven Giftwirkungen des Phenylhydroxylamin, eines Blutgiftes, habe ich im Anschluß an einen von mir beobachieten Vergiftungsfall und auf Grund von Tierversuchen früher schon Mitteilungen gemacht. ${ }^{1}$ ) Wie dieser Stoff auch Gewebsveränderungen zu erzeugen vermag, konnte ich kürzlich an mir selbst erfahren, nachdem ich in eine wässerige Lösung eines Alkaloidsalzes kristallinisches Phenylhydroxylamin im Ueberschub eingetragen und die Masse bis zur Lösung erwärmt hatte. Mit dem Daumen der linken Hand hatte ich dag Reagierglas verschlossen und die heibe Lösung wiederholt um. geschüttelt.

Nach 11/2 Tagen machte sich an dem Daumen, soweit er in die Oeffnung des Reagierglases eingedrückt und durch die Elüssigkeit benetzt worden war, eine leichte, härtliche Schwellung bemerk.

1) L. Lew in, Archiv farr experimentelle Pathologie und Pharmakologie Bd. $35,1893$. 
bar, und an dem geschwollenen Teile empfand ich, nur bei Berührung, ein ganz eigenartiges, vielleicht als stechend und brennend zu bezeichnendes Gefühl, das nach $2 \%$ Tagen zu einer unangenehmen Schmerzempfindung anwuchs. Auch der leichteste Druck an dem erkrankten Teile war jetzt so unerträglich, daß man annehmen mußte, es habe sich in der jetzt stärker, polsterartig geschwollenen Haut eine Blase gebildet.

Nach vier Tagen erschien an der dem Zeigefinger zugekehrten Seite dieses Daumens eine unregelmäßige Erhabenheit von etwa $1 \frac{1}{9} \mathrm{~cm}$ Länge und $1 \mathrm{~cm}$ Breite, die sich in den nächsten Tagen rötete und ebenso wie die primär erkrankte Daumenpartie auf Berührung eine schmerzende Empfindung entstehen lieB. Ein stärkerer Druck machte die Stelle braunrot. Diese Farbe wich nun nicht mehr. In den nächstfolgenden sieben Tagen entwickelten sich ähnliche, zum Teil wenig erhabene, unregelmäßige, etwas indurierte, braunrote Flecke in der Hohlhand, zuvörderst an der Hautfalte zwischen Daumen und Zeigefinger und an den Hügelchen über den Fingerkommissuren und dann auch an den Fingern. An jedem von ihnen entstanden in verschiedenen Zeiträumen Flecke der angegebenen Art, und zwar am Zeigefinger an der radialen Seite seines ersten Feldes gleich unterhalb der obersten Hautfurche und zum Teil auf seinen Rücken übergreifend; ferner an dem zweiten bis fünften Finger je ein oder auch zwei Flecke, zum Teil in der Mitte ihrer drei Felder, zum Teil radialwärts gelegen. Am stärksten schmerzempfindlich und geschwollen waren die Tastpolster, als wenn wie am Daumen so auch hier in der Tiefe der Cutis Blasen säßen.

Die Hand war völlig unbrauchbar und blieb in dem geschilderten Zustande, bis auf eine stärker werdende Dunkelrotfärbung der erkrankten Teile, 14 Tage unverändert. Nur zweimal während dieser Zeit empfand ich ziehende Schmerzen bis zur Ellenbeuge, ohne daB sich jedoch irgendwelche Veränderung an den Armlymphgefäßen zeigte. Weder kalte Umschläge, noch Einpackungen, noch Fetteinreibung, die trotz der dadurch erzeugten starken Schmerzen des Versuches wegen gemacht wurden, schufen eine Veränderung. Erst durch mehrstiindige, tägliche Seifenbäder erfolgte eine Besserung der Schmerzempfindung, der Schwellung etc. Allmählich schwanden auch die Flecke. Thre Spuren und zeitweise juckende Empfindung machten sich noch acht Wochen nach dem Beginn der Hautvergiftung bemerkbar.

\section{Epikritische Bemerkungen.}

Die hier niedergelegte Erfahrung hat eine praktische Bedeutung, insofern sie lehrt, dal dem Phenylhydroxylamin, das ein starkes Reduktionsmittel darstellt, unangenehme örtliche Wirkungen zukommen, besonders wenn konzentrierte Lösungen die Haut berühren. Darüber hinaus gibt sie mir den Anlab, auf das eigenartige Vorrücken der Hautveränderung von einer ganz umschriebenen Stelle des Daumens aus auf andere Teile der Hand aufmerksam zu machen. Daß es sich hier nicht um eine resorptive Wirkung im eigentlichen Sinne des Wortes handelt, scheint mir sicher zu sein; denn wenn das Gift von der Haut des linken Daumens aus in die Blutgefäße eingedrungen wäre, dann hätte es vielleicht auch an andern Teilen der linken Hand oder in irgend einer Ausdehnung auch an weiteren Körperstellen in kurzer Zeit eine Wirkung entfalten müssen. Eine solche fehlte hier aber vollständig. Der ent. zündliche Prozeß rückte nur an der linken Hand langsam vor und lokalisierte sich so eigenartig, dab man an eine Verbreitung des Giftes durch die oberflächlichen Lymphwege der Haut, den Arcus lymphaticus volaris und seine Ausläufer, denken mub. Auf der Höhe der Hautvergiftung spiegelte die Gesamtheit der Veränderungen so ziemlich das Bild dieser anatomischen Einrichtung wider.

Bei der Anwendung mancher Arzneimittel, z. B. von Antisepticis, sind Hautveränderungen auch entfernt vom Anwendungsort beobachtet worden, freilich nicht in so eigenartiger Anordnung wie die eben beschriebenen, aber immerhin doch so, dab die Vermutung nahegelegt wurde, daf sie ihr Entstehen einer Fortbewegung des gewebsreizenden Giftes in den oberflächlichen Lymphbahnen der Haut verdankten. Ich erinnere z. B. an die nach Jodoform entstehenden Hautveränderungen, die nach einiger Zeit auch in nähcrer oder weiterer Entfernung von der Anwendungsstelle auftreten können. Jede andere Erklärung für das Entstehen derartiger, zumal diskontinuierlicher Veränderungen, und besonders die Heranziehung eines Nerveneinflusses, verlangt schwerer verständliche Annahmen als die hier gegebene. Sie ist es, die schließlich auch für die Ausbildung der Lymphangitis in Frage kommt. Hierbei kann man davon Abstand nehmen auf das Irrtümliche der sehr weit verbreiteten Meinung besonders hinzuweisen, daß diese Erkrankung stets die Folge einer örtlichen Infektion sei und in letzter Reihe nur durch das Findringen von pathogenen Pilzen in die Lymphspalten und ihr Weiterwandern bedingt sei. Auch Gifte können das gleiche Leiden hervorrufe n. So erzeugt z. B. der Schlange n bi gelegentlich örtliche Hautveränderungen und im Anschluß daran Iy mphangitis und Lymphadenitis, nicht etwa weil die beiBende Schlange Mikroorganismen mit ihren Giftzähnen in die Wunde gebracht hat, sondern weil den Eiweifistoffen des Schlangengiftes wie manchen andern Proteïnen eine entzündungserregende Fähigkeit zukommt. Deswegen können auch, z. B. bci der puerperalen Sepsis, Gelenkund Gliederschwellungen so durch zerfallenes Körpereiweiß entstehen, wie das Einbringen von fremdem, zersetztem Eiweiß in der Form der "Heilsera" sie gelegentlich hervorrufen.

Die näheren Bedingungen sind nicht bekannt, unter denen gewisse Stoffe vorzugsweise in den Lymphwegen der Haut vorrücken, wenn diese chemisch oder thermisch oder mechanisch verletzt worden ist. Es können hierfür in Frage kommen: die Beschaffenheit des Giftes und die Art seines Eindringens. Obschon eiweißartige Gifte, einschließlich von Tiergiften und Krankheitsgiften, die in die Haut gelangen, auffallend häufig in ihr für eine gewisse Zeit einen Weg zurückzulegen scheinen, ehe sie nach längerem oder kürzerem Verweilen in den Lymphdrüsen in die Blutbahn gelangen, so gibt es doch auch genug andersartige Stoffe, die sich ähnlich verhalten und, z. B. in Gewerbebetrieben, gerade durch diese Eigenschaft Hauterkrankungen veranlassen. Auffallend ist es, dab stark reduzierende Substanzen in dieser Gruppe häufig angetroffen werden. Wichtiger als die Beschaffenheit der Gifte scheint mir der Ort ihres Eindringens zu sein. Es sind gewöhnlich die oberflächlichsten Kontinuitätstrennungen der Haut, die schon selbst längst geheilt sein können, von denen aus die Lymphangitis als ein Zeichen der Weiterwanderung des Giftes sich entwickelt. So kann das Gift eines feinsten, in das Epidermislager gedrungenen Raupenhaares wirken, und so vermag Phenylhydroxylamin oder ein anderer Körper, der die Epidermis langsam chemisch verändert, seinen Weg in irgend einer Form in die freigelegten Lymphspalten zu nehmen.

Wie die weitere Fortbewegung sich vollzieht, ist wie so sehr vieles Wissenswerte gerade auf dem Gebiete des Lymphgefäßsystems nicht genügend erforscht. Der Druck, unter dem Lymphflüssigkeiten sich bewegen, ist sehr klein und die Bewegung sehr langsam. Selbst die am Ductus thoracicus eines Hundes ausfließende Lymphmenge beträgt für jede Stunde nur etwa $27 \mathrm{ccm}$ und ist vom Aortendruck so gut wie unabhängig. Demnach kann man ermessen, wie langsam fremde, reaktive chemische Stoffe in den Lymphcapillaren vorrücken und wie lange Zeit vergehen kann, ehe durch Deponierung des reaktiven Giftes an irgend welchen Stellen der Hautlymphwege Veränderungen entstehen. Ein äußerer Druck auf die Eintrittsstelle des Giftes oder Muskelkontraktionen können die Verbreitung des Giftes, seine eventuelle gewebsverändernde Wirkung und seinen Uebertritt in die Blutbahn beschleunigen. Es wird in jedem solchen Falle zu erwägen sein, ob völlige Ruhe des vergifteten Teiles oder dessen aktive oder passive Bewegung für die Heilung des örtlichen Leidens, bzw. für die Entgiftung vorteilhafter ist. Für die meisten Fälle wird wohl das letztere anzunehmen sein. 